\title{
A Comparative Study of Adaptive Registration Methods for Two Dimensional Gel Electrophoresis Images
}

\author{
Huamei Xin, Yuemin Zhu, Longfei Wu
}

\begin{abstract}
Two-dimensional gel electrophoresis (2DGE) images play a major role in techniques for protein separation. The registration of $2 \mathrm{DGE}$ images is considered as one of key elements in protein identification. This paper proposes a single adaptive registration scheme for $2 D G E$ images. Within the scheme, three registration methods based on the combined use of global linear affine and local nonlinear thin-plate spline (TPS), Demons, B-spline algorithms are explored and compared. The results show that the method using multi-resolution B-spline is preferred.
\end{abstract}

\section{INTRODUCTION}

$T^{\infty}$ wo-dimensional gel electrophoresis (2DGE) is currently the leading proteomic technique for analyzing, visualizing and quantifying the proteins expressed in a biological system at a given time. Originally introduced by Patrick O'Farrell for analyzing Escherichia coli proteins in 1975 [1], 2DGE separates protein first in the direction of isoelectric points (pI), then in that of molecular weight (MW), thus generating a two-dimensional (2D) images, called 2DGE or gels images, in which the separated proteins appear as blob spots with various gray levels and sizes on a bright background.

Comparing 2DGE images enables to detect changes in protein expressions or even to identify new proteins. However, before performing such comparison, there should be geometrical correspondence between the images to be compared. This is a registration problem in terms of image processing. Therefore, registration of $2 \mathrm{DGE}$ images is an important step in bioinformatics study of proteins. Due to complex physical and chemical processes, the locations of the same protein spots in different gels images may differ in a both globally and locally different manner, so an adaptive registration scheme is needed to take into account such global

Manuscript received March 30, 2007.

Huamei Xin and Yuemin Zhu are with the CREATIS-LRMN, CNRS, UMR 5220, Villeurbanne, F-69621, France; Inserm, U630, Villeurbanne, F-69621, France; INSA-Lyon, Villeurbanne, F-69621, France; Université de Lyon, Lyon, F-69003, France; Université Lyon 1, Villeurbanne, F-69622, France.(e-mail: huamei.xin@creatis.insa-lyon.fr).

Longfei $\mathrm{Wu}$ is with the CNRS UPR9043, Laboratoire de Chimie Bactérienne, F-13402, Marseille, France. and local displacements of 2DGE images. Previously proposed methods for electrophoresis images registration can be classified into three categories: landmark-based, intensity-based, and landmark-intensity based. Since registration based on intensity or landmark alone is often not sufficiently accurate for registering 2DGE images, the method based on both intensity and landmark information was proposed [2] [3], showing that this category of methods significantly improves registration accuracy.

However, in the field of medical imaging, it was shown that, for the same medical images to be registered, different registration methods leads to different registrations results [4] [5] [6]. Meanwhile, our experiments on 2DGE images also revealed that different registration methods led to significantly varying results. The purpose of this paper is to propose a single adaptive registration scheme for $2 \mathrm{DGE}$ images. Within the scheme, three registration methods based on the combined use of global linear affine and local nonlinear thin-plate spline (TPS), Demons and B-spline algorithms are explored and compared.

\section{METHODS}

Registration of 2DGE images consists in finding corresponding protein spots in two input images. To do that, an adaptive registration scheme is investigated.

Firstly, since geometric distortions mainly result from global changes of the electric field in gel images, we perform a global multi-resolution affine registration. The affine transform is a linear transformation that maps lines into lines. It can be used to represent translations, rotations, anisotropic scaling, shearing or any combination of them. A registration based on the affine transform may be more effective when applied to remove the major global misalignment. This affine registration step is carried out by fine tuning the scale parameters of the optimizer. With the multi-resolution process, the registration is first performed at the coarsest level using the images at the first level of the fixed and moving image pyramids, then the transform parameters determined by the registration are used to initialize the registration at the next finer level using images from the second level of the pyramids. Although this global affine step is not extremely accurate, it is a good initial registration.

Then, since, after global affine registration, there still exist 
local deformations presented by combinational factors, a nonlinear local registration method is required. To do this, we introduce three different nonlinear registration methods based respectively on the TPS, Demons, and B-spline models.

Assume that $E_{\text {total }}$ represents a general energy function for the above three nonlinear methods, which is to be minimized during the registration. It can be described as following:

$$
E_{\text {total }}=w_{s} E_{\text {sim }}+w_{r} E_{\text {reg }}+w_{c} E_{\text {constra int }}
$$

where $E_{\text {sim }}$ is a similarity energy aiming at finding the correspondences, $E_{\text {reg }}$, a regularization energy, used to smooth displacement fields, and $E_{\text {constraint, }}$ a constraint term, served to incorporate forward and backward transformations.

The three weighting parameters, $w_{s}, w_{r}, w_{c}$, which represent the weight of the similarity term, the regularizing term and the constraint term respectively, aim to adjust the relative strength of the three different energies.

For nonlinear registration based on the TPS [7], the first term measures the sum of the quadratic Euclidean distances between two images, the second term controls the smoothness of the transformation, the third term gives the harmony of the forward and backward transformations, and the parameters $w_{s}, w_{r}$, and $w_{c}$ weight the relevance of the similarity energy with respect to the regularization energy and the constraint energy.

With regards to nonlinear registration based on Demons [8], $E_{\text {sim }}$ is provided by the intensity similarity measure such as the sum of squared differences (SSD) because 2DGE images that we deal with are nearly binary, $E_{\text {reg }}$ determines the smoothness of the displacement field through smoothing the force field using a Gaussian filter, and by $E_{\text {constraint }}$, both the gradient of the fixed image and that of the moving image are taken into account. The choice of the three parameters $w_{s}, w_{r}$ and $w_{c}$ make it possible to adjust the effect of these three different terms.

Concerning nonlinear registration based on B-spline [9], in our particular application to 2DGE images, we use SSD, and the Limited memory Broyden-Fletcher-Goldfarb-Shanno (LBFGS) optimizer is used to optimize the transformation parameters. $w_{s}, w_{r}, w_{c}$ define the relative importance of each term of the energy function.

In our present study, a multi-resolution strategy is used in the three nonlinear registration methods. This makes it reasonable to use a coarse and fast interpolator in the lower resolution levels and switch to a high-quality but slow interpolator in the final resolution level, thus making the registration more robust.

\section{EXPERIMENTS AND RESULTS}

The proposed registration scheme was evaluated using 2D gel electrophoresis images of the Escherichia coli proteome, and the experiments were carried out as the following: firstly, we simulated global and local distortions, and performed the registration between the original and distorted images. Then, the proposed method was tested with 5 pairs of real gel images. All original images were acquired in the Bacterial chemistry laboratory, Marseille, France.

\section{A. Experiment with simulated images}

The simulated pairs of images are such as those shown in Fig. 1. An original image was used as the fixed image, and the moving image to be registered with respect to the original image was simulated by applying global and local distortions on the original image.

Fig. 2 shows the results of registering the simulated images given in Fig. 1, using our scheme based on respectively the TPS $\left(w_{s}=1, w_{r}=1, w_{c}=1\right)$, Demons $\left(w_{s}=1, w_{r}=2, w_{c}=1\right)$, and B-spline $\left(w_{s}=1, w_{r}=1, w_{c}=1\right)$ methods. The left column represents the images after registration, and the right column the difference images between the original and registered images. To more quantitatively assess the registration accuracy, we counted the number of matched spots in the two images. The results are given in Table I, it is observed that the registration based on B-spline gives much better result.

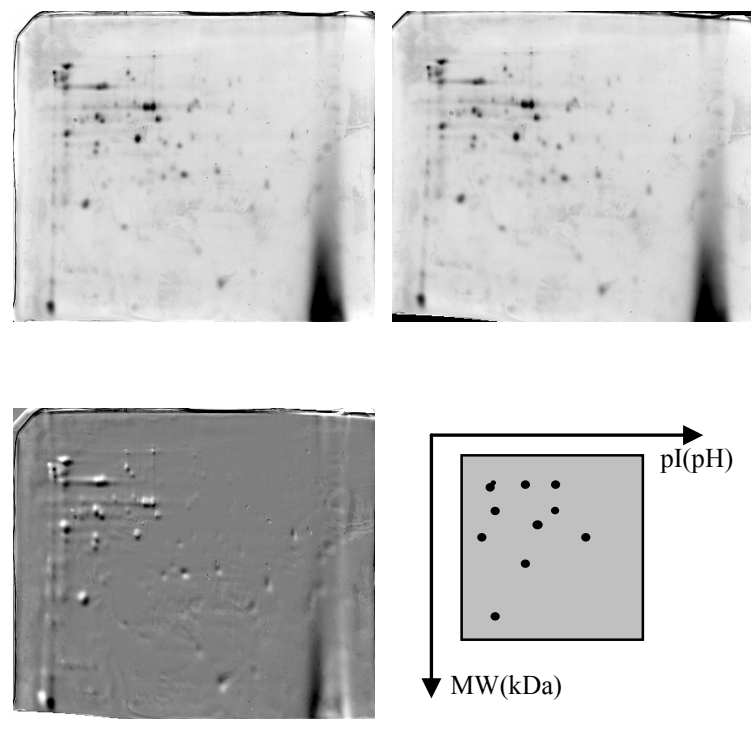

Fig. 1. Simulated pair of images before registration. From top to down, left to right: original image, simulated image with global and local distortions, difference image before registration, schematic 2DGE image. 


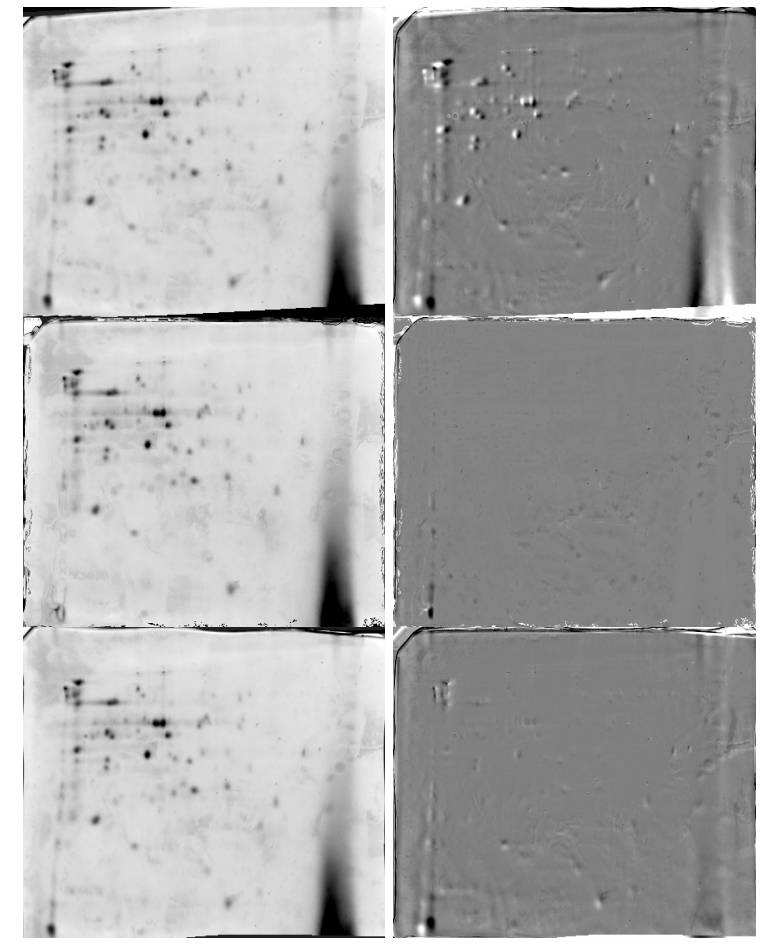

Fig. 2. Results of registering the simulated images using our scheme based on respectively the TPS, Demons and B-spline methods. Left column: images after registration. Right column: difference images between the original and registered images.

TABLE I

QUANTITATIVE EVALUATION OF REGISTRATION RESULTS ON SIMULATED IMAGES

\begin{tabular}{cc}
\hline \hline & $\begin{array}{c}\text { Percentage of correct } \\
\text { matched spots }\end{array}$ \\
Registration based on TPS & $84.5 \%$ \\
Registration based on Demons & $93.6 \%$ \\
Registration based on B-spline & $94.9 \%$ \\
\hline \hline
\end{tabular}

\section{B. Experiment with pairs of real gel images}

The used real gel images have ground truth in the sense that the corresponding spot pairs have been established by biologists. An example of such real gel images before registration is shown in Fig. 3, in which one original image is used as the fixed image, and the other as the moving image. These two images represent the protein content of a solution under different biological conditions, that is to say, the images in a pair do not show the same information. We are also interested in knowing the difference in spot number and position between the two images, since it contains very useful information about Escherichia coli. It is seen that the difference image has great values, showing that there is no geometrical correspondence between the fixed and moving images before registration.

The results of registering the real gel images using our scheme based on respectively the TPS $\left(w_{s}=1, w_{r}=1, w_{c}=1\right)$, Demons $\left(w_{s}=1, w_{r}=2, w_{c}=1\right)$, and B-spline $\left(w_{s}=1\right.$, $w_{r}=1, w_{c}=1$ ) are shown in Fig. 4. The quantitative assessment of the registration accuracy is given in Table II. Both the difference image in Fig. 4 and quantitative results in Table II show that the registration based on B-spline gives much better results.

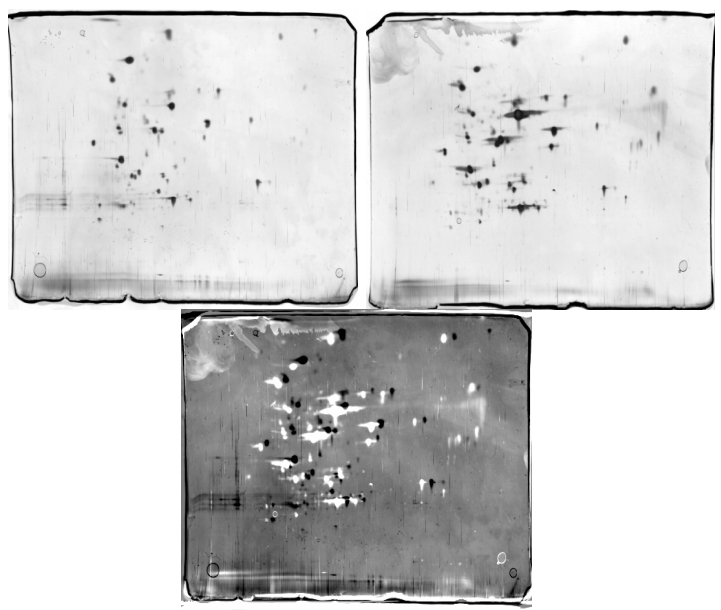

Fig. 3. Illustration of real gel images before registration. From top to down, left to right: original fixed image, original moving image, difference image between the fixed and moving images.

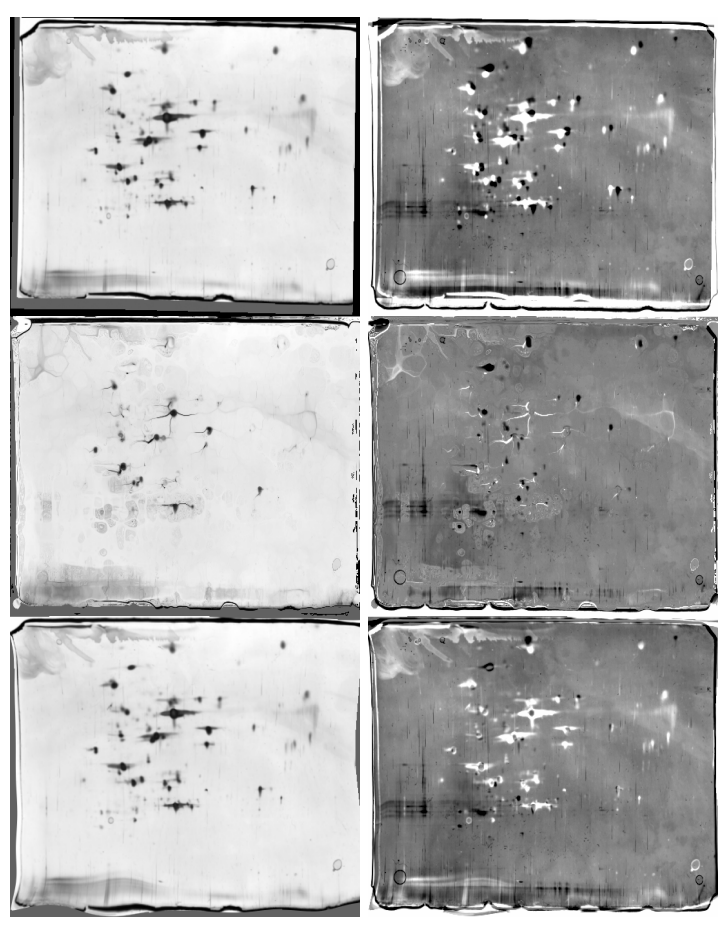

Fig. 4. Results of registering real gel images using our scheme based on respectively the TPS, Demons and B-spline methods. Left column: images after registration. Right column: difference images between the original and registered images. 
TABLE II

QUANTITATIVE EVALUATION OF REGISTRATION

RESULTS ON THE REAL GEL IMAGES

SHOWN IN FIG. 3

\begin{tabular}{cc}
\hline \hline & $\begin{array}{c}\text { Percentage of correct } \\
\text { matched spots }\end{array}$ \\
Registration based on TPS & $83.2 \%$ \\
Registration based on Demons & $92.7 \%$ \\
Registration based on B-spline & $96.1 \%$ \\
\hline \hline
\end{tabular}

Table III shows registration results on other five pairs of real gel images. They also show that B-spline based registration leads to better results.

TABLE III

AVERAGE QUANTITATIVE EVALUATION RESULTS OF THE REGISTRATION FOR FIVE PAIRS OF REAL IMAGES

\begin{tabular}{cc}
\hline \hline & $\begin{array}{c}\text { Percentage of correct } \\
\text { matching spots }\end{array}$ \\
Registration based on TPS & $82.4 \%$ \\
Registration based on Demons & $93.4 \%$ \\
Registration based on B-spline & $95.3 \%$ \\
\hline \hline
\end{tabular}

\section{Discussions AND CONCULUSion}

The results obtained with both simulated and real gel images show that the registration based on TPS or Demons was not ideal. The main reasons probably are:

For TPS algorithm, when the control points are irregularly spaced, large errors may occur for points away from the control points due to radially symmetric logarithmic functions. Also, and when logarithmic basis functions monotonically increase to cover the entire image domain, the transformation becomes global and cannot well adapt to local deformations.

In the case of Demons algorithm, although it is a model adaptable to local deformations, it depends largely on gray level information of the images by assuming that the intensity is constant with time, whereas two corresponding spots in 2DGE images may have rather different gray levels, which could result in the problem of the consistency.

Concerning B-spline algorithm, the interpolation between nodes has the local support characteristic, and only voxels within a radius of about two node spacing are affected by translation of that node. All that is consistent with the characteristic of the deformations in 2D gels images.

Hence, in view of the fact the three methods present little difference in computation time, we conclude that the registration method based on the combined use of global linear affine and local nonlinear B-spline algorithms is preferred for the registration of 2DGE images. It is worth noting that experiments taking into account the "smile" deformation in gels images could further improve registration results in the case of B-spline method [10]. It would then be useful to consider such deformation priors also in the other two methods. In a more general manner, further in-depth study about the general characteristic deformations of gels images would be interesting in the future. Finally, to get more complete insights into the three methods, it would be necessary to other criteria.

\section{ACKNOWLEDGMENT}

We would like to thank Prof. Robert Goutte for helpful discussions.

\section{REFERENCES}

[1] P. H. O'Farrell, "High resolution two-dimensional electrophoresis of proteins," J. of Biological Chemistr, vol.250, pp. 4007-4021, 1975.

[2] X. Y. Wang, D. D. Feng, H. Hong, "Novel elastic registration for 2-D medical and gel protein images," Conferences in Research and Practice in Information Technology, vol.19, 2003.

[3] K. Rohr, P. Cathier, S. Wörz, "Elastic registration of electrophoresis images using intensity information and point landmarks," Pattern Recognition, vol.37, pp.1035-1048, 2004.

[4] T. Popa, L. Ibáñez, K. Cleary, K. H. Wong, "ITK implementation of deformation methods for time-varing(4D) imaging data," Medical Imaging 2006: Visualization, Image-Guided Procedures, and Display, vol.6141, pp. 750-759, 2006.

[5] L. Zagorchev, A. Goshtasby, "A comparative study of transformation functions for nonrigid image registration," IEEE Transaction on Image Processing, vol.15, pp.529-538, 2006.

[6] T. Rohlfing. "Transformation model and constraints cause bias in statistics on deformation fields," MICCAI 2006, LNCS 4190, pp.207-214, 2006.

[7] F. L. Bookstein, "Principal warps: thin-plate splines and the decomposition of deformations," IEEE Trans. Pattern Anal. Mach. Intell., vol. 11, pp. 567-585, 1989.

[8] J. P. Thirion, "Fast non-rigid matching of 3D medical images," Technical Report 2547, INRIA, Sophia Antipolis, France, 1995.

[9] M. Unser, A. Aldroubi, M. Eden, "Fast B-spline transforms for continuous image representation and interpolation," IEEE Trans. Pattern Anal. Mach. Intell., vol.13, no.3, pp.277-285, 1991.

[10] C.O.S. Sorzano, P. Thévenaz, M. Unser, "Elastic Registration of Biological Images Using Vector-Spline Regularization," IEEE Trans. Biomedical Engineering, vol.52, no.4, pp.652-663, 2005. 